a marche. Alors ...

\title{
THEOREMES DE CONNEXITE ET VARIETES ABELIENNES
}

\author{
Olivier Debarre $(*)$
}

Cet article est consacré à la démonstration d'un théorème de connexité pour les variétés abéliennes, ainsi qu'à l'exposition de quelques corollaires. Les résultats analogues pour les espaces projectifs et leurs conséquences sont connus depuis un moment déjà $([\mathrm{FH}],[\mathrm{FL}],[\mathrm{F} 1])$ et fournissent un guide qu'il n'y a qu'à suivre.

Le théorème principal énonce que si on se donne une variété abélienne $\mathrm{X}$ et deux morphismes $\mathrm{V} \rightarrow \mathrm{X}$ et $\mathrm{W} \rightarrow \mathrm{X}$, alors, sous certaines hypothèses, assez contraignantes mais indispensables, $\mathrm{V} \times_{\mathrm{X}} \mathrm{W}$ est connexe. Les conséquences de ce résultat sont nombreuses.

Par exemple, on montre que le groupe fondamental algébrique d'une sous-variété normale d'une variété abélienne simple $\mathrm{X}$, de dimension $>\frac{1}{2} \operatorname{dim}(\mathrm{X})$, est isomorphe à celui de $\mathrm{X}$ (cela découle de résultats antérieurs ([S]) lorsque $\mathrm{V}$ est lisse).

On étend aussi certains résultats de Nori $([\mathrm{N}])$ : si D est un diviseur dans une variété abélienne $\mathrm{X}$, dont aucune composante n'est une sous-variété abélienne, et qui est à croisements normaux en dehors d'un fermé de codimension 2 dans $\mathrm{D}$, alors le noyau du morphisme surjectif $\pi_{1}(\mathrm{X}-\mathrm{D}) \rightarrow \pi_{1}(\mathrm{X})$ est abélien libre de type fini, et l'on détermine son rang.

Des résultats analogues à ceux de [L1] sont aussi obtenus, comme par exemple que le groupe fondamental algébrique d'une variété normale $\mathrm{V}$ qui est revêtement de degré $\leq \operatorname{dim}(\mathrm{V})$ d'une variété abélienne simple $\mathrm{X}$, est isomorphe à celui de $\mathrm{X}$. Lorsque $\operatorname{dim}(\mathrm{V}) \geq 2$, la même conclusion subsiste pour un revêtement qui induit une bijection ensembliste au-dessus d'une courbe, comme par exemple un revêtement cyclique, sans condition sur son degré.

On termine avec une conjecture, analogue d'un résultat démontré pour les espaces projectifs par Lazarsfeld. Nous renvoyons le lecteur au $\S 8$ pour son énoncé, un peu technique.

\section{Rappels et notations}

Dans tout cet article, on travaille sur un corps algébriquement clos $k$ de caractéristique nulle. Une variété est un schéma projectif réduit de type fini sur $k$, pas nécessairement irréductible, mais qu'on supposera toujours non vide.

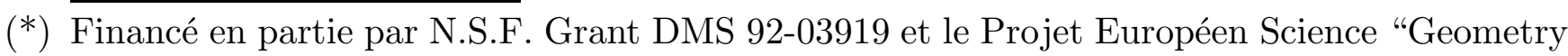
of Algebraic Varieties", Contract no. SCI-0398-C (A). 
Le groupe fondamental algébrique d'un schéma est défini dans [G1], Exp. V ( $c f$. aussi [Mu1], p. 169). Comme nous ne considérerons que des espaces connexes, nous écrirons simplement $\pi_{1}^{\text {alg }}(\mathrm{X})$ pour le groupe fondamental algébrique d'une variété connexe $\mathrm{X}$.

Lorsque $k=\mathbf{C}$, le groupe $\pi_{1}^{\text {alg }}(\mathrm{X})$ est le complété du groupe fondamental topologique $\pi_{1}(\mathrm{X})$ pour la topologie des sous-groupes d'indices finis ([G1], Exp. XII, cor. 5.2).

(1.1) Soit Pic(X) le schéma de Picard de X. Pour tout entier $n>0$, on note ${ }_{n} \operatorname{Pic}(\mathrm{X})$ le noyau de la multiplication par $n$ dans $\operatorname{Pic}(\mathrm{X})$; il existe un isomorphisme ([G1], Exp. XI, p. 305) :

$$
\operatorname{Hom}\left(\pi_{1}^{\text {alg }}(\mathrm{X}), \mathbf{Z} / n \mathbf{Z}\right) \simeq{ }_{n} \operatorname{Pic}(\mathrm{X})
$$

On notera $\mathrm{Pic}^{0}(\mathrm{X})$ la composante neutre de $\mathrm{Pic}(\mathrm{X})$. Lorsque $\mathrm{X}$ est normal, $\operatorname{Pic}^{0}(\mathrm{X})_{\text {red }}$ est une variété abélienne ([G2], Exp. VI, cor. 3.2).

Soient $\mathrm{V}$ une variété connexe et $f: \mathrm{V} \rightarrow \mathrm{X}$ un morphisme. Il induit alors un morphisme $f_{*}: \pi_{1}^{\text {alg }}(\mathrm{V}) \rightarrow \pi_{1}^{\text {alg }}(\mathrm{X})([\mathrm{G} 1]$, Exp. V, p. 142) et :

(1.2) $f_{*}$ est surjectif si et seulement si pour tout revêtement étale connexe $\mathrm{X}^{\prime} \rightarrow \mathrm{X}$, le revêtement étale $\mathrm{V} \times_{\mathrm{X}} \mathrm{X}^{\prime} \rightarrow \mathrm{V}$ est connexe ([G1], Exp. V, prop. 6.9);

(1.3) $f_{*}$ est injectif si et seulement si pour tout revêtement étale $\mathrm{V}^{\prime} \rightarrow \mathrm{V}$, il existe un revêtement étale $\mathrm{X}^{\prime} \rightarrow \mathrm{X}$ et un $\mathrm{V}$-morphisme d'une composante connexe de $\mathrm{V} \times_{\mathrm{X}} \mathrm{X}^{\prime}$ dans $\mathrm{V}^{\prime}$. C'est le cas en particulier si tout revêtement étale connexe de $\mathrm{V}$ est isomorphe à un revêtement du type $\mathrm{V} \times{ }_{\mathrm{X}} \mathrm{X}^{\prime} \rightarrow \mathrm{V}$ ([G1], Exp. V, cor. 6.8 et remarque 6.12);

(1.4) si $f$ est étale, alors $f_{*}$ est injectif ([G1], Exp. V, p. 142).

(1.5) On suppose de plus $\mathrm{X}$ normal. Lorsque $f_{*}$ est surjectif, le morphisme induit $f_{0}^{*}: \mathrm{Pic}^{0}(\mathrm{X})_{\text {red }} \rightarrow \operatorname{Pic}^{0}(\mathrm{~V})_{\text {red }}$ est un plongement. En effet, pour tout entier $n>0$, il ressort de (1.1) que les morphismes induits $f_{n}^{*}:{ }_{n} \operatorname{Pic}(\mathrm{X}) \rightarrow{ }_{n} \operatorname{Pic}(\mathrm{V})$ sont surjectifs, de sorte que $\operatorname{Ker}\left(f_{0}^{*}\right)$ est sans torsion, donc nul. Si de plus $\mathrm{V}$ est normal et $f_{*}$ bijectif, alors $f_{0}^{*}$ est un isomorphisme (même démonstration).

(1.6) Supposons $\mathrm{X}$ (géométriquement) unibranche, c'est-à-dire que pour tout point $x$ de $\mathrm{X}$, le normalisé de l'anneau local $\mathcal{O}_{\mathrm{X}, x}$ a un seul idéal maximal. Alors, si $\mathrm{X}^{\prime} \rightarrow \mathrm{X}$ est un revêtement étale connexe, $\mathrm{X}^{\prime}$ est irréductible et unibranche ([GD1], 17.5.7). D'autre part, si $\tilde{\mathrm{X}} \rightarrow \mathrm{X}$ est la normalisation; le morphisme induit $\pi_{1}^{\text {alg }}(\tilde{\mathrm{X}}) \rightarrow \pi_{1}^{\text {alg }}(\mathrm{X})$ est un isomorphisme ([G1], Exp. IX, th. 4.10).

(1.7) Soit maintenant $\mathrm{X}$ une variété abélienne. Alors $\pi_{1}^{\text {alg }}(\mathrm{X})$ est isomorphe à $\hat{\mathrm{Z}}^{2 \operatorname{dim}(\mathrm{X})}$ ([G1], Exp. XI, th. 2.1). Il résulte de [Mu1], p. 147 et 171, que deux variétés abéliennes isogènes ont des groupes fondamentaux algébriques isomorphes. Lorsque $k=\mathbf{C}$, le groupe $\pi_{1}(\mathrm{X})$ est isomorphe à $\mathbf{Z}^{2 \operatorname{dim}(\mathrm{X})}$.

(1.8) Soient $\mathrm{V}$ une variété et $f: \mathrm{V} \rightarrow \mathrm{X}$ un morphisme. On appelera factorisation de 
$f$ la donnée d'une variété abélienne $\mathrm{X}^{\prime}$, d'une isogénie $q: \mathrm{X}^{\prime} \rightarrow \mathrm{X}$ et d'un morphisme $f^{\prime}: \mathrm{V} \rightarrow \mathrm{X}^{\prime}$ tels que $f=q f^{\prime}$. On dira que $f$ est minimal si, pour toute factorisation, $q$ est un isomorphisme. Supposons que $f(\mathrm{~V})$ engendre $\mathrm{X}$. Le noyau du morphisme induit $f^{*}: \operatorname{Pic}^{0}(\mathrm{X}) \rightarrow \operatorname{Pic}(\mathrm{V})$ est alors fini et correspond à une isogénie $q: \mathrm{X}^{\prime} \rightarrow \mathrm{X}$ à travers laquelle $f$ se factorise. La factorisation correspondante de $f$ est alors minimale.

Enfin, si $\mathrm{V}$ est unibranche et $f: \mathrm{V} \rightarrow \mathrm{X}$ minimal, et si $\eta: \tilde{\mathrm{V}} \rightarrow \mathrm{V}$ est la normalisation de $\mathrm{V}$, le morphisme composé $\tilde{f}=f \eta$ est aussi minimal. En effet, soit $\tilde{\mathrm{V}} \rightarrow \mathrm{X}^{\prime} \rightarrow \mathrm{X}$ une factorisation de $\tilde{f}$. Le morphisme $\eta$ se factorise alors en $\tilde{\mathrm{V}} \stackrel{g}{\rightarrow} \mathrm{V} \times_{\mathrm{X}} \mathrm{X}^{\prime} \rightarrow \mathrm{V}$. Soit $\mathrm{V}^{\prime}$ la composante connexe de $\mathrm{V} \times_{\mathrm{X}} \mathrm{X}^{\prime}$ qui contient $g(\tilde{\mathrm{V}})$. Par (1.6), $\mathrm{V}^{\prime}$ est irréductible, donc égal à $g(\tilde{\mathrm{V}})$. Le morphisme étale $\mathrm{V}^{\prime} \rightarrow \mathrm{V}$ est donc un isomorphisme; $f$ se factorise alors à travers $q$, qui est donc un isomorphisme.

(1.9) Pour énoncer notre résultat principal (théorème 4.5) avec des hypothèses minimales, nous aurons besoin de la définition suivante. Soit $\mathrm{X}$ une variété abélienne et soient $\mathrm{V}$ et $\mathrm{W}$ deux sous-variétés de $\mathrm{X}$. On dit que $(\mathrm{V}, \mathrm{W})$ remplit (resp. remplit strictement) $\mathrm{X}$ si, pour toute sous-variété abélienne propre $\mathrm{K}$ de $\mathrm{X}$, on a :

$$
\operatorname{dim}(\pi(\mathrm{V}))+\operatorname{dim}(\pi(\mathrm{W})) \geq \operatorname{dim}(\mathrm{X} / \mathrm{K})
$$

(resp. $>$ ), où $\pi: \mathrm{X} \rightarrow \mathrm{X} / \mathrm{K}$ est la surjection canonique. Par exemple, (V,X) remplit strictement $\mathrm{X}$ si et seulement si $\mathrm{V}$ engendre $\mathrm{X}$. Lorsque $\mathrm{X}$ est simple, ou encore lorsque toute courbe contenue dans $\mathrm{V}$ engendre $\mathrm{X}$, le couple $(\mathrm{V}, \mathrm{W})$ remplit (resp. remplit strictement) $\mathrm{X}$ si et seulement si $\operatorname{dim}(\mathrm{V})+\operatorname{dim}(\mathrm{W}) \geq \operatorname{dim}(\mathrm{X})$ (resp. $>$ ).

Afin de ne pas trop alourdir le texte, nous avons préféré énoncer les différents corollaires avec des hypothèses plus faibles (mais plus parlantes). Le lecteur pourra rétablir facilement les énoncés optimaux.

\section{Sous-variétés lisses}

En ce qui concerne les sous-variétés lisses d'une variété abélienne, Sommese a obtenu dans une série d'articles, qui culmine avec [So], des résultats très complets dont voici un échantillon :

Théorème 2.1. (Sommese) - Soit $\mathrm{X}$ une variété abélienne complexe simple et soient $\mathrm{V}$ et $\mathrm{W}$ deux sous-variétés lisses de $\mathrm{X}$. On suppose $\mathrm{W}$ connexe et $\mathrm{V} \cap \mathrm{W}$ non vide. Alors $\pi_{q}(\mathrm{~W}, \mathrm{~V} \cap \mathrm{W})=0$ pour $q \leq \min (\operatorname{dim}(\mathrm{W}), \operatorname{dim}(\mathrm{V})+1)-\operatorname{codim}(\mathrm{V})$.

On verra plus loin (théorème 3.1 ) que $\mathrm{V} \cap \mathrm{W}$ est non vide dès que $\operatorname{dim}(\mathrm{W}) \geq \operatorname{codim}(\mathrm{V})$, c'est-à-dire dès que la borne supérieure sur $q$ est $\geq 0$.

Corollaire 2.2. - Soient $\mathrm{X}$ une variété abélienne complexe simple et $\mathrm{V}$ une sous-variété lisse connexe de $\mathrm{X}$. Alors :

(i) le morphisme induit $\pi_{1}(\mathrm{~V}) \rightarrow \pi_{1}(\mathrm{X})$ est surjectif pour $\operatorname{dim}(\mathrm{V}) \geq \frac{1}{2} \operatorname{dim}(\mathrm{X})$ et est un isomorphisme pour $\operatorname{dim}(\mathrm{V})>\frac{1}{2} \operatorname{dim}(\mathrm{X})$, 
(ii) le morphisme de restriction $\operatorname{Pic}(\mathrm{X}) \rightarrow \operatorname{Pic}(\mathrm{V})$ est injectif pour $\operatorname{dim}(\mathrm{V})>\frac{1}{2} \operatorname{dim}(\mathrm{X})$ et est un isomorphisme pour $\operatorname{dim}(\mathrm{V}) \geq 1+\frac{1}{2} \operatorname{dim}(\mathrm{X})$.

Remarque 2.3. Soit $\mathrm{C}$ une courbe projective lisse connexe de genre $g$, soit JC sa jacobienne et soit $\mathrm{W}_{d}(\mathrm{C})$ la sous-variété de JC qui paramètre les classes d'équivalence de diviseurs effectifs de degré $d$ sur $\mathrm{C}$. Lorsque $d<g$, pour tout point $x$ de $\mathrm{C}$, le diviseur de Weil $x+\mathrm{W}_{d-1}(\mathrm{C})$ ne peut être la restriction à $\mathrm{W}_{d}(\mathrm{C})$ d'un diviseur de $\mathrm{JC}$, pour des raisons numériques. Pour $\mathrm{C}$ générique et $d<1+\frac{1}{2} g$, JC est simple, $\mathrm{W}_{d}(\mathrm{C})$ est lisse, mais la restriction $\mathrm{Pic}(\mathrm{JC}) \rightarrow \operatorname{Pic}\left(\mathrm{W}_{d}(\mathrm{C})\right)$ n'est donc pas surjective. La deuxième borne du corollaire 2.2.(ii) est donc la meilleure possible. On remarquera qu'on retrouve aussi ainsi le fait bien connu que $\mathrm{W}_{d}(\mathrm{C})$ n'est pas lisse pour $g>d \geq 1+\frac{1}{2} g$. En général, on peut montrer que la restriction $\mathrm{Pic}(\mathrm{JC}) \rightarrow \operatorname{Pic}\left(\mathrm{W}_{d}(\mathrm{C})\right)$ est un isomorphisme si et seulement si $\mathrm{W}_{d}(\mathrm{C})$ est singulier, donc en particulier lorsque $d \geq 1+\frac{1}{2} g$. Je pense d'ailleurs que le (ii) du corollaire devrait rester vrai pour $\mathrm{V}$ normal.

\section{Sous-variétés quelconques}

Les premiers résultats sur l'intersection de deux sous-variétés quelconques d'une variété abélienne précèdent en fait ceux de Sommese. Ils sont dus à Barth, qui a démontré dans [B1] le théorème suivant lorsque $\mathrm{X}$ est simple.

Théorème 3.1. - Soit $\mathrm{X}$ une variété abélienne et soient $\mathrm{V}$ et $\mathrm{W}$ deux sous-variétés irréductibles de $\mathrm{X}$ telles que $(\mathrm{V}, \mathrm{W})$ remplisse $\mathrm{X}$ (cf. (1.9)). Alors $\mathrm{V} \cap \mathrm{W} \neq \emptyset$.

Remarques 3.2. 1) La conclusion ne subsiste pas en général si $\mathrm{V}$ ou $\mathrm{W}$ est réductible : si $\mathrm{Y}$ est une variété abélienne simple de dimension $\geq 2$, E une courbe elliptique, $\mathrm{C}$ une courbe dans $\mathrm{Y}$ ne contenant pas l'origine, et $e$ un point non nul de $\mathrm{E}$, alors $\mathrm{V}=\mathrm{C} \times\{0\}$ et $\mathrm{W}=\{0\} \times \mathrm{E} \cup \mathrm{Y} \times\{e\}$ remplissent $\mathrm{X}=\mathrm{Y} \times \mathrm{E}$, mais ne se rencontrent pas.

2) Le début de la démonstration montre que lorsque toute courbe contenue dans $\mathrm{V}$ engendre $\mathrm{X}$, la conclusion subsiste quelle que soit la caractéristique de $k$. De plus, si $\mathrm{V}_{1}, \ldots, \mathrm{V}_{r}$ sont des sous-variétés de $\mathrm{X}$ vérifiant $\sum_{i=1}^{r} \operatorname{codim}_{\mathrm{X}}\left(\mathrm{V}_{i}\right) \leq \operatorname{dim}(\mathrm{X})$ et si toute courbe contenue dans $\mathrm{V}_{1}$ engendre $\mathrm{X}$, alors $\bigcap_{i=1}^{r} \mathrm{~V}_{i} \neq \emptyset$.

Démonstration du théorème. On raisonne par récurrence sur la dimension de $\mathrm{X}$. Si V ne rencontre pas $\mathrm{W}$, le point 0 n'est pas dans l'image $(\mathrm{V}-\mathrm{W})$ du morphisme $m: \mathrm{V} \times \mathrm{W} \rightarrow \mathrm{X}$ défini par $m(v, w)=v-w$. Ce dernier n'est donc pas surjectif. Soit $a=v-w$ un point générique, donc lisse, de $(\mathrm{V}-\mathrm{W})$. Il existe une sous-variété $\mathrm{F}$ de $\mathrm{X}$ de dimension $>0$, contenant 0 , telle que :

$$
m^{-1}(a)=\{(v+e, w+e) \mid e \in \mathrm{F}\} .
$$

On a alors $(a+\mathrm{F}-\mathrm{F}) \subset(\mathrm{V}-\mathrm{W})$, de sorte que $\mathrm{T}_{a}(a+\mathrm{F}-\mathrm{F}) \subset \mathrm{T}_{a}(\mathrm{~V}-\mathrm{W})$. Soit $<\mathrm{F}>$ la sous-variété abélienne de $\mathrm{X}$ engendrée par $\mathrm{F}$.

Lemme 3.3. - On a $\mathrm{T}_{0}(\mathrm{~F}-\mathrm{F})=\mathrm{T}_{0}<\mathrm{F}>$. 
Démonstration. En utilisant le théorème de Lefschetz, on se ramène à montrer que si une courbe $\mathrm{C}$ (peut-être réductible) engendre $\mathrm{X}$, alors $\mathrm{T}_{0}(\mathrm{C}-\mathrm{C})=\mathrm{T}_{0} \mathrm{X}$. On note $\mathcal{G}: \mathrm{C}_{\text {reg }} \rightarrow \mathbf{P} \mathrm{T}_{0} \mathrm{X}$ l'application de Gauss. Pour tout $x \in \mathrm{C}_{\text {reg }}$, on a :

$$
\mathcal{G}(x)=\mathbf{P T}_{0}(\mathrm{C}-x) \subset \mathbf{P} \mathrm{T}_{0}(\mathrm{C}-\mathrm{C}),
$$

de sorte qu'il suffit de montrer que l'image de $\mathcal{G}$ n'est pas contenue dans un hyperplan. Cela résulte du fait que la restriction $\mathrm{H}^{0}\left(\mathrm{X}, \Omega_{\mathrm{X}}^{1}\right) \rightarrow \mathrm{H}^{0}\left(\mathrm{C}_{\mathrm{reg}}, \Omega_{\mathrm{C}_{\text {reg }}}^{1}\right)$ est injective.

On a donc $\mathrm{T}_{a}(a+<\mathrm{F}>) \subset \mathrm{T}_{a}(\mathrm{~V}-\mathrm{W})$. Le point $a$ étant générique, on se convainc rapidement que pour $x$ dans un voisinage de a dans $(\mathrm{V}-\mathrm{W})$, la sous-variété abélienne correspondante (c'est-à-dire $<p r_{1} m^{-1}(x)>$ ) reste égale à $<\mathrm{F}>$. Pour $x$ générique dans $(\mathrm{V}-\mathrm{W})$, on a donc $\mathrm{T}_{x}(x+<\mathrm{F}>) \subset \mathrm{T}_{x}(\mathrm{~V}-\mathrm{W})$.

Lemme 3.4. - Soient $\mathrm{X}$ une variété abélienne, $\mathrm{Z}$ une sous-variété irréductible de $\mathrm{X}$ et $\mathrm{K}$ une sous-variété abélienne de $\mathrm{X}$. On suppose que, pour z générique dans $\mathrm{Z}$, on a $\mathrm{T}_{z}(z+\mathrm{K}) \subset \mathrm{T}_{z} \mathrm{Z}$. Alors, $\mathrm{Z}+\mathrm{K}=\mathrm{Z}$.

Démonstration. Comme on est en caractéristique 0 , il existe un point $(z, k)$ lisse sur $\mathrm{Z} \times \mathrm{K}$ en lequel la différentielle du morphisme d'addition $\mathrm{Z} \times \mathrm{K} \rightarrow(\mathrm{Z}+\mathrm{K})$ est surjective. L'image de cette différentielle est par hypothèse $\mathrm{T}_{z+k}(\mathrm{Z}+k)$, de sorte que $\operatorname{dim}(\mathrm{Z}+\mathrm{K})=\operatorname{dim}(\mathrm{Z})$, ce qui prouve le lemme.

Il est clair que les images de $\mathrm{V}$ et $\mathrm{W}$ dans $\mathrm{X} /<\mathrm{F}>$ remplissent $\mathrm{X} /<\mathrm{F}>$. Le lemme entraîne alors $\mathrm{V}-\mathrm{W}+<\mathrm{F}>=\mathrm{V}-\mathrm{W}$ et l'hypothèse de récurrence $(\mathrm{V}-\mathrm{W}) \cap<\mathrm{F}>\neq \emptyset$. On en déduit $0 \in(\mathrm{V}-\mathrm{W})$, ce qui termine la démonstration.

Corollaire 3.5. - Soient $\mathrm{X}$ une variété abélienne simple et $\mathrm{V}$ une sous-variété de $\mathrm{X}$ vérifiant $\operatorname{dim}(\mathrm{V}) \geq \frac{1}{2} \operatorname{dim}(\mathrm{X})$. Alors le morphisme induit $\pi_{1}^{\mathrm{alg}}(\mathrm{V}) \rightarrow \pi_{1}^{\mathrm{alg}}(\mathrm{X})$ est surjectif. Lorsque $k=\mathbf{C}$, il en est de même du morphisme $\pi_{1}(\mathrm{~V}) \rightarrow \pi_{1}(\mathrm{X})$.

Démonstration. Soit $\mathrm{X}^{\prime} \rightarrow \mathrm{X}$ une isogénie. Par (1.2), il s'agit de montrer que la sousvariété $\mathrm{V} \times_{\mathrm{X}} \mathrm{X}^{\prime}$ de $\mathrm{X}^{\prime}$ est connexe. Cela résulte du fait que ses composantes irréductibles sont toutes de même dimension que $\mathrm{V}$, donc se rencontrent deux à deux par le théorème. Lorsque $k=\mathbf{C}$, soit $\Gamma$ le $\mathbf{Z}$-module libre $\pi_{1}(\mathrm{X})(1.7)$. Pour tout entier $n>0$, le morphisme composé $\pi_{1}(\mathrm{~V}) \rightarrow \Gamma \rightarrow \Gamma / n \Gamma$ est alors surjectif. Le conoyau $\mathrm{Q}$ de $\pi_{1}(\mathrm{~V}) \rightarrow \Gamma$ est alors un groupe abélien de type fini qui vérifie $\mathrm{Q}=n \mathrm{Q}$ pour tout $n>0$. Il est donc nul, ce qui termine la démonstration du corollaire.

Remarques 3.6. 1) L'hypothèse optimale est ici que (V, V) remplit X (cf. (1.9)).

2) Sous les hypothèses du corollaire, on a en particulier que le morphisme d'inclusion $\mathrm{V} \hookrightarrow \mathrm{X}$ est minimal au sens de (1.8). 
Dans un second article [B2], semble-t-il peu connu, Barth s'est ensuite intéressé à la connexité de l'intersection de deux sous-variétés, soit dans une variété abélienne, soit dans l'espace projectif, obtenant ainsi des résultats dont il faudra attendre plus de dix ans pour qu'ils soient généralisés et appliqués par Fulton et Hansen dans le cas de l'espace projectif ( $c f$. [F1] et [FL] pour des compte-rendus). A ma connaissance, le cas des variétés abéliennes est resté ouvert. Il s'avère que beaucoup des résultats obtenus dans l'espace projectif restent valables avec quelques modifications.

\section{Théorèmes de connexité}

Commençons par le résultat technique suivant, dont la démonstration suit des idées de Mumford telles qu'elles sont exposées dans [FL], p. 42.

Proposition 4.1. - Soient $\mathrm{X}$ une variété abélienne, $\tilde{\mathrm{V}}, \tilde{\mathrm{W}}$ et $\mathrm{Y}$ des variétés irréductibles normales, $f: \tilde{\mathrm{V}} \rightarrow \mathrm{X}$ et $g: \tilde{\mathrm{W}} \rightarrow \mathrm{X}$ deux morphismes et $\pi: \mathrm{Y} \rightarrow \tilde{\mathrm{V}} \times \tilde{\mathrm{W}}$ un morphisme étale. On suppose que $(f(\tilde{\mathrm{V}}), g(\tilde{\mathrm{W}}))$ remplit strictement $\mathrm{X}$. Soit $m: \mathrm{X} \times \mathrm{X} \rightarrow \mathrm{X}$ le morphisme défini par $m(x, y)=x-y$. Alors le morphisme $\mathrm{M}=m \circ(f, g) \circ \pi$ se factorise en $\mathrm{M}: \mathrm{Y} \stackrel{h}{\rightarrow} \mathrm{X}^{\prime} \stackrel{q}{\rightarrow} \mathrm{X}$, où $\mathrm{X}^{\prime}$ est une variété abélienne, $q$ une isogénie, et où les fibres de $h$ sont connexes.

Démonstration. Elle est basée sur le lemme suivant :

Lemme 4.2 - Sous les hypothèses de la proposition, si D est une sous-variété irréductible de $\mathrm{X}$ et $\mathrm{Z}$ une composante irréductible de $\mathrm{M}^{-1}(\mathrm{D})$ telle que, pour z générique dans $\mathrm{Z}$, on ait $\mathrm{T}_{z} \mathrm{M}\left(\mathrm{T}_{z} \mathrm{Z}\right) \subset \mathrm{T}_{\mathrm{M}(z)} \mathrm{D}$, alors $\mathrm{D}$ n'est pas un diviseur.

Démonstration. Procédant par récurrence sur la dimension de $\mathrm{X}$, on raisonne par l'absurde en supposant que $\mathrm{D}$ est un diviseur. Posons $\mathrm{V}^{\prime}=f(\tilde{\mathrm{V}})$ et $\mathrm{W}^{\prime}=g(\tilde{\mathrm{W}})$. On note encore $m$ l'application $\mathrm{V}^{\prime} \times \mathrm{W}^{\prime} \rightarrow \mathrm{X}$ induite par $m$. Soit $z$ un point générique de $\mathrm{Z}$, on note $\pi(z)=(\tilde{v}, \tilde{w}), \quad v^{\prime}=f(\tilde{v}), \quad w^{\prime}=g(\tilde{w})$ et $a=\mathrm{M}(z)=v^{\prime}-w^{\prime}$. Comme $\operatorname{dim}\left(\mathrm{V}^{\prime}\right)+\operatorname{dim}\left(\mathrm{W}^{\prime}\right)>\operatorname{dim}(\mathrm{X})$, il existe une sous-variété $\mathrm{F}$ de $\mathrm{X}$ de dimension $>0$, contenant 0 , telle que :

$$
m^{-1}(a)=\left\{\left(v^{\prime}+e, w^{\prime}+e\right) \mid e \in \mathrm{F}\right\} .
$$

Le lieu où l'application $\tilde{\mathrm{V}} \rightarrow \mathrm{V}^{\prime}$ induite par $f$ n'est pas lisse, est un fermé propre de $\tilde{\mathrm{V}}$. Il en est de même pour le morphisme $\tilde{\mathrm{W}} \rightarrow \mathrm{W}^{\prime}$ induit par $g$. Comme $\mathrm{Z}$ est un diviseur et que $\mathrm{T}$ est étale, on peut donc supposer par exemple que l'image de la différentielle de $(f, g) \circ \pi$ en $z$ contient $\mathrm{T}_{v^{\prime}}\left(\mathrm{V}^{\prime}\right) \times\{0\}$. Il en résulte que l'on a, pour tout point $e$ de $\mathrm{F}$ :

$$
a+\mathrm{T}_{e} \mathrm{~F} \subset-w^{\prime}+\mathrm{T}_{v^{\prime}} \mathrm{V}^{\prime} \subset \mathrm{T}_{z} \mathrm{M}\left(\mathrm{T}_{z} \mathrm{Z}\right) \subset \mathrm{T}_{a} \mathrm{D}
$$

Si $\mathrm{K}$ est la sous-variété abélienne de $\mathrm{X}$ (de dimension $>0$ ) engendrée par $\mathrm{F}$, il en résulte que $\mathrm{T}_{a}(a+\mathrm{K}) \subset \mathrm{T}_{a} \mathrm{D}$. Comme dans la démonstration du théorème 3.1, on en déduit que pour $x$ générique dans $\mathrm{D}$, on a $\mathrm{T}_{x}(x+\mathrm{K}) \subset \mathrm{T}_{x} \mathrm{D}$. Le lemme 3.4 entraîne alors $\mathrm{D}+\mathrm{K}=\mathrm{D}$. Lorsque $\mathrm{X}$ est simple (et en particulier si $\operatorname{dim}(\mathrm{X})=1$ ), on en déduit 
une contradiction. Sinon, l'image de $\mathrm{D}$ dans $\mathrm{X} / \mathrm{K}$ est encore un diviseur et l'hypothèse de récurrence entraîne encore une contradiction. Ceci termine la démonstration du lemme.

Revenons à la démonstration de la proposition. Notons $\mathrm{Y} \stackrel{h}{\rightarrow} \mathrm{X}^{\prime} \stackrel{q}{\rightarrow} \mathrm{X}$ la factorisation de Stein de $\mathrm{M}$, où $\mathrm{X}^{\prime}$ est normal, $q$ fini surjectif, et où les fibres de $h$ sont connexes, de dimension $>0$. Si $q$ est ramifié, on choisit une composante $\mathrm{D}^{\prime}$ de son lieu de ramification; par le théorème de pureté $([\mathrm{Z}]), \mathrm{D}=q\left(\mathrm{D}^{\prime}\right)$ est un diviseur de $\mathrm{X}$. En un point générique $x^{\prime}$ de $\mathrm{D}^{\prime}$, le morphisme $\mathrm{D}^{\prime} \rightarrow \mathrm{D}$ induit par $q$ est lisse, de sorte que l'image de la différentielle $\mathrm{T}_{x^{\prime}} q: \mathrm{T}_{x^{\prime}} \mathrm{X}^{\prime} \rightarrow \mathrm{T}_{q\left(x^{\prime}\right)} \mathrm{X}$ contient $\mathrm{T}_{q\left(x^{\prime}\right)} \mathrm{D}$. Comme $\mathrm{T}_{x^{\prime}} q$ n'est pas surjective, son image est donc $\mathrm{T}_{q\left(x^{\prime}\right)} \mathrm{D}$. Prenant pour $\mathrm{Z}$ une composante irréductible de $h^{-1}\left(\mathrm{D}^{\prime}\right)$, on obtient une contradiction avec le lemme 4.2. Il en résulte que $q$ est non ramifié, donc étale ([G1], Exp. I, th. 9.5), et que $\mathrm{X}^{\prime}$ est une variété abélienne ([Mu1], p. 167).

Le théorème suivant généralise un résultat de Barth ([B2], Satz 2).

Théorème 4.3. - Soient $\mathrm{X}$ une variété abélienne simple, $\mathrm{V}$ une variété irréductible, $f: \mathrm{V} \rightarrow \mathrm{X}$ un morphisme et $\mathrm{W}$ une sous-variété irréductible de $\mathrm{X}$. On suppose que $\operatorname{dim}(f(\mathrm{~V}))+2 \operatorname{dim}(\mathrm{W}) \geq 2 \operatorname{dim}(\mathrm{X})$. Alors $f^{-1}(\mathrm{~W})$ est connexe et, si $\mathrm{V}$ est de plus unibranche, le morphisme induit $\pi_{1}^{\text {alg }}\left(f^{-1}(\mathrm{~W})\right) \rightarrow \pi_{1}^{\text {alg }}(\mathrm{V})$ est surjectif.

Remarque 4.4. La première conclusion du théorème ne subsiste pas lorsqu'on suppose seulement $\operatorname{dim}(f(\mathrm{~V}))+\operatorname{dim}(\mathrm{W})>\operatorname{dim}(\mathrm{X})$, comme le montre l'exemple suivant dû̀ à Barth $([\mathrm{B} 2])$ : soit $\mathrm{X}^{\prime}$ une variété abélienne simple de dimension 7 et $\mathrm{W}^{\prime}$ une sous-variété de $\mathrm{X}^{\prime}$ lisse connexe de dimension 3 . Il existe un sous-groupe fini $\mathrm{G}$ non trivial de $\mathrm{X}^{\prime}$ tel que $\mathrm{W}^{\prime} \cap \mathrm{W}_{\gamma}^{\prime}$ soit vide pour tout élément non nul $\gamma$ de $\mathrm{G}$. On pose $\mathrm{X}=\mathrm{X}^{\prime} / \mathrm{G}$ et on note $\mathrm{W}$ l'image de $\mathrm{W}^{\prime}$ dans $\mathrm{X}$ par la projection canonique. Alors $\mathrm{W}$ est lisse et il existe une sousvariété $\mathrm{V}$ irréductible de $\mathrm{X}$ de dimension 5 (intersection complète de deux hypersurfaces), telle que $\mathrm{V} \cap \mathrm{W}$ soit réunion de $\operatorname{Card}(\mathrm{G})$ courbes disjointes.

Démonstration du théorème. On note $\eta_{\mathrm{V}}: \tilde{\mathrm{V}} \rightarrow \mathrm{V}$ et $\eta_{\mathrm{W}}: \tilde{\mathrm{W}} \rightarrow \mathrm{W}$ les normalisations. Soit $\tilde{\mathrm{V}} \times \tilde{\mathrm{W}} \stackrel{h}{\rightarrow} \mathrm{X}^{\prime} \stackrel{q}{\rightarrow} \mathrm{X}$ la factorisation du morphisme $(\tilde{v}, \tilde{w}) \mapsto\left(f \eta_{\mathrm{V}}(\tilde{v})-g \eta_{\mathrm{W}}(\tilde{w})\right)$ fournie par la proposition 4.1. On note $\mathrm{G}$ le noyau de $q$, on choisit un point $\tilde{v}_{0}$ de $\tilde{\mathrm{V}}$, un point $\tilde{w}_{0}$ de $\tilde{\mathrm{W}}$, et on définit des morphismes $f^{\prime}: \tilde{\mathrm{V}} \rightarrow \mathrm{X}^{\prime}$ et $g^{\prime}: \tilde{\mathrm{W}} \rightarrow \mathrm{X}^{\prime} \operatorname{par} f^{\prime}(\tilde{v})=h\left(\tilde{v}, \tilde{w}_{0}\right)$ et $g^{\prime}(\tilde{w})=-h\left(\tilde{v}_{0}, \tilde{w}\right)$. L'image du morphisme $(\tilde{v}, \tilde{w}) \mapsto h(\tilde{v}, \tilde{w})-f^{\prime}(\tilde{v})+g^{\prime}(\tilde{w})$ est alors contenue dans l'ensemble fini $q^{-1}\left(q h\left(\tilde{v}_{0}, \tilde{w}_{0}\right)\right)$, donc est réduite au seul point $\alpha=-h\left(\tilde{v}_{0}, \tilde{w}_{0}\right)$. On a donc :

$$
h(\tilde{v}, \tilde{w})=f^{\prime}(\tilde{v})-g^{\prime}(\tilde{w})+\alpha .
$$

Remarquons que $f^{-1}(\mathrm{~W})$ est l'image par $\eta_{\mathrm{V}} p r_{1}$ de $(q h)^{-1}(0)=h^{-1}(\mathrm{G})$. Il suffit donc de montrer que $p r_{1} h^{-1}(\mathrm{G})$ est connexe. Pour tout $\gamma \in \mathrm{G}$, l'ensemble $p r_{1} h^{-1}(\gamma)=f^{\prime-1}\left(g^{\prime}(\tilde{\mathrm{W}})+\gamma-\alpha\right)$ est connexe. Par la remarque 3.2.2), l'intersection de $f^{\prime}(\tilde{\mathrm{V}}), \quad g^{\prime}(\tilde{\mathrm{W}})+\gamma-\alpha$ et $g^{\prime}(\tilde{\mathrm{W}})-\alpha$ est non vide, de sorte que $p r_{1} h^{-1}(\gamma)$ rencontre $p r_{1} h^{-1}(0)$. Cela entraîne que $p r_{1} h^{-1}(\mathrm{G})$, donc aussi $f^{-1}(\mathrm{~W})$, est connexe, ce qui termine 
la démonstration du premier point du théorème.

Supposons maintenant $\mathrm{V}$ unibranche. Soit $\pi: \mathrm{V}^{\prime} \rightarrow \mathrm{V}$ un revêtement étale connexe; $\mathrm{V}^{\prime}$ est alors irréductible (1.6). Par (1.2), il s'agit de montrer que $f^{-1}(\mathrm{~W}) \times_{\mathrm{V}} \mathrm{V}^{\prime}$ est connexe. Or cela résulte du premier point, puisque cet ensemble est $(f \pi)^{-1}(\mathrm{~W})$.

Le théorème suivant (analogue de [FL], th. 4.1) montre que, moyennant des hypothèses supplémentaires sur les singularités des variétés, on peut obtenir des résultats plus forts.

Théorème 4.5. - Soient $\mathrm{X}$ une variété abélienne, $\mathrm{V}$ et $\mathrm{W}$ deux variétés irréductibles et $f: \mathrm{V} \rightarrow \mathrm{X}$ et $g: \mathrm{W} \rightarrow \mathrm{X}$ deux morphismes. On suppose que $\mathrm{V}$ est unibranche, que $f$ est minimal au sens de (1.8), et que $(f(\mathrm{~V}), g(\mathrm{~W}))$ remplit strictement $\mathrm{X}$ (cf. (1.9)). Alors $\mathrm{V} \times_{\mathrm{X}} \mathrm{W}$ est connexe. De plus, si on note $\iota$ le plongement $\mathrm{V} \times_{\mathrm{X}} \mathrm{W} \hookrightarrow \mathrm{V} \times \mathrm{W}$ et $\mathrm{M}: \mathrm{V} \times \mathrm{W} \rightarrow \mathrm{X}$ le morphisme $(v, w) \mapsto(f(v)-g(w))$, alors :

$$
\pi_{1}^{\text {alg }}\left(\mathrm{V} \times_{\mathrm{X}} \mathrm{W}\right) \stackrel{\iota_{*}}{\longrightarrow} \pi_{1}^{\mathrm{alg}}(\mathrm{V} \times \mathrm{W}) \stackrel{\mathrm{M}_{*}}{\longrightarrow} \pi_{1}^{\mathrm{alg}}(\mathrm{X}) \rightarrow 0
$$

est un complexe exact en $\pi_{1}^{\mathrm{alg}}(\mathrm{X})$. Si on suppose de plus $\mathrm{W}$ unibranche, il est exact.

Remarque 4.6. La première conclusion ne subsiste pas lorsqu'on ne fait aucune hypothèse sur les singularités de V, comme le montre l'exemple de Barth (remarque 4.4).

Démonstration du théorème. Soient $\eta_{\mathrm{V}}: \tilde{\mathrm{V}} \rightarrow \mathrm{V}$ et $\eta_{\mathrm{W}}: \tilde{\mathrm{W}} \rightarrow \mathrm{W}$ les normalisations et $\tilde{\mathrm{V}} \times \tilde{\mathrm{W}} \stackrel{h}{\rightarrow} \mathrm{X}^{\prime} \stackrel{q}{\rightarrow} \mathrm{X}$ la factorisation de $\mathrm{M} \circ\left(\eta_{\mathrm{V}}, \eta_{\mathrm{W}}\right)$ fournie par le lemme 4.3. Par (1.8), le morphisme $\tilde{\mathrm{V}} \rightarrow \mathrm{V} \rightarrow \mathrm{X}$ est encore minimal, de sorte que $q$ est un isomorphisme; $\mathrm{V} \times_{\mathrm{X}} \mathrm{W}$ est alors l'image de $h^{-1}(0)$ par un morphisme continu, donc est connexe. Cela montre le premier point.

Pour le second, on remarque que $\mathrm{V} \times_{\mathrm{X}} \mathrm{W}$ est la fibre en 0 de $\mathrm{M}$, de sorte qu'on a bien un complexe. Ensuite, si $\mathrm{X}^{\prime} \rightarrow \mathrm{X}$ est une isogénie, ce qui précède montre que $\mathrm{V} \times_{\mathrm{X}} \mathrm{X}^{\prime}$ est connexe; le morphisme $\pi_{1}^{\text {alg }}(\mathrm{V}) \rightarrow \pi_{1}^{\text {alg }}(\mathrm{X})$ est donc surjectif par (1.2). Il en est alors de même de $\pi_{1}^{\text {alg }}(\mathrm{V} \times \mathrm{W}) \rightarrow \pi_{1}^{\mathrm{alg}}(\mathrm{X})$.

Pour montrer l'exactitude en $\pi_{1}^{\text {alg }}(\mathrm{V} \times \mathrm{W})$, on peut supposer $\mathrm{V}$ et $\mathrm{W}$ normales par (1.6) et (1.8). On se donne un revêtement étale connexe $\pi: \mathrm{Y} \rightarrow \mathrm{V} \times \mathrm{W}$, on pose $\mathrm{Z}=\mathrm{V} \times_{\mathrm{X}} \mathrm{W}$ et on suppose que le revêtement étale induit $\pi^{-1}(\mathrm{Z}) \rightarrow \mathrm{Z}$ a une section. Par [G1], Exp. V, prop. 6.11, il s'agit de montrer qu'il existe un revêtement étale $\mathrm{X}^{\prime} \rightarrow \mathrm{X}$ et un morphisme $(\mathrm{V} \times \mathrm{W}) \times_{\mathrm{X}} \mathrm{X}^{\prime} \rightarrow \mathrm{Y}$ au-dessus de $\mathrm{V} \times \mathrm{W}$. Comme $\mathrm{Y}$ est irréductible normale 
(1.6), la proposition 4.1 fournit un diagramme commutatif :

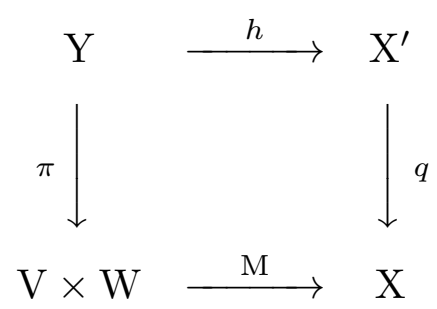

où les fibres de $h$ sont connexes et où $q$ est une isogénie, dont on note $\mathrm{K}$ le noyau et $k$ le degré. Les morphismes $\pi$ et $h$ se factorisent à travers un morphisme $\rho: \mathrm{Y} \rightarrow(\mathrm{V} \times \mathrm{W}) \times_{\mathrm{X}} \mathrm{X}^{\prime}$ qui, puisque $(\mathrm{V} \times \mathrm{W}) \times_{\mathrm{X}} \mathrm{X}^{\prime}$ est connexe (par le premier point, puisque $\mathrm{V} \times \mathrm{W}$ est unibranche), est étale surjectif ([G1], Exp. V, prop. 3.5); on peut donc écrire $h=h^{\prime} \rho$ et $\pi=\pi^{\prime} \rho$. D'autre part, $\pi^{-1}(\mathrm{Z})=h^{-1}(\mathrm{~K})$ a $k$ composantes connexes $\mathrm{Y}_{1}, \ldots, \mathrm{Y}_{k}$ et les composantes connexes de $\pi^{\prime-1}(\mathrm{Z})=h^{\prime-1}(\mathrm{~K})$ sont $\rho\left(\mathrm{Y}_{1}\right), \ldots, \rho\left(\mathrm{Y}_{k}\right)$, avec $\rho^{-1}\left(\rho\left(\mathrm{Y}_{i}\right)\right)=\mathrm{Y}_{i}$ pour tout $i$. Or par hypothèse, un des revêtements étales $\rho^{-1}\left(\rho\left(\mathrm{Y}_{i}\right)\right) \rightarrow \rho\left(\mathrm{Y}_{i}\right)$ a une section; c'est donc un isomorphisme par [G1], Exp. I, cor. 5.3. Comme $\mathrm{V} \times \mathrm{W}$ et $\rho^{-1}\left(\rho\left(\mathrm{Y}_{i}\right)\right)$ sont connexes, cela prouve que $\rho$ est un isomorphisme ([G1], Exp. I, cor. 10.10) et son inverse est le morphisme cherché.

Corollaire 4.7. - Soient X une variété abélienne simple, V une variété irréductible unibranche et $f: \mathrm{V} \rightarrow \mathrm{X}$ un morphisme non constant, minimal au sens de (1.8). Alors, le morphisme induit $\pi_{1}^{\mathrm{alg}}(\mathrm{V}) \rightarrow \pi_{1}^{\mathrm{alg}}(\mathrm{X})$ est surjectif. Lorsque $k=\mathbf{C}$, il en est de même $d u$ morphisme $\pi_{1}(\mathrm{~V}) \rightarrow \pi_{1}(\mathrm{X})$.

Démonstration. La première assertion découle du théorème, puisque $(\mathrm{V}, \mathrm{X})$ remplit strictement $\mathrm{X}(c f .(1.9))$. Lorsque $k=\mathbf{C}$, on raisonne comme dans la démonstration du cor. 3.5.

\section{Groupe fondamental des sous-variétés}

Comme dans [FL] $\S 5$, nous appliquons le théorème de connexité à l'étude du groupe fondamental des sous-variétés d'une variété abélienne. Le théorème suivant est l'analogue de [FL], th. 5.1.

Théorème 5.1. - Soient $\mathrm{X}$ une variété abélienne simple, $\mathrm{V}$ une variété irréductible unibranche et $f: \mathrm{V} \rightarrow \mathrm{X}$ un morphisme non ramifié. On suppose que $\operatorname{dim}(f(\mathrm{~V}))>\frac{1}{2} \operatorname{dim}(\mathrm{X})$. Alors il existe une variété abélienne $\mathrm{X}^{\prime}$, une isogénie $q: \mathrm{X}^{\prime} \rightarrow \mathrm{X}$ et un plongement $f^{\prime}: \mathrm{V} \rightarrow \mathrm{X}^{\prime}$ tels que $f=q f^{\prime}$.

Démonstration. Soit $f: \mathrm{V} \stackrel{f^{\prime}}{\rightarrow} \mathrm{X}^{\prime} \stackrel{q}{\rightarrow} \mathrm{X}$ une factorisation de $f$ avec $f^{\prime}$ minimal (1.8). On suit la démonstration de $[\mathrm{FL}]$, p. 46 : le théorème 4.5 implique que $\mathrm{V} \times{ }_{\mathrm{X}^{\prime}} \mathrm{V}$ est 
connexe. Comme $f^{\prime}$ est non ramifié, la diagonale $\Delta_{\mathrm{V}}$ est ouverte dans $\mathrm{V} \times \times_{\mathrm{X}^{\prime}} \mathrm{V}$. Etant aussi fermée, on a $\Delta_{\mathrm{V}}=\mathrm{V} \times_{\mathrm{X}^{\prime}} \mathrm{V}$, de sorte que $f^{\prime}$ est bijective. On conclut en remarquant qu'un morphisme non ramifié bijectif est un plongement ([GD2], 8.11.5 et [GD1], 17.2.6).

On en déduit la généralisation suivante de la deuxième partie du corollaire 2.2 :

Corollaire 5.2. - Soient $\mathrm{X}$ une variété abélienne simple et $\mathrm{V}$ une sous-variété irréductible unibranche de $\mathrm{X}$. On suppose que $\operatorname{dim}(\mathrm{V})>\frac{1}{2} \operatorname{dim}(\mathrm{X})$. Alors, le morphisme induit $\pi_{1}^{\mathrm{alg}}(\mathrm{V}) \rightarrow \pi_{1}^{\mathrm{alg}}(\mathrm{X})$ est un isomorphisme.

Démonstration. Par la remarque 3.6.2), l'inclusion $\mathrm{V} \hookrightarrow \mathrm{X}$ est minimale. Soit $\pi: \mathrm{V}^{\prime} \rightarrow \mathrm{V}$ un revêtement étale connexe; $\mathrm{V}$ étant irréductible et unibranche, il en est de même de $\mathrm{V}^{\prime}$ (1.6). Le théorème précédent s'applique au morphisme non ramifié $\mathrm{V}^{\prime} \stackrel{\pi}{\rightarrow} \mathrm{V} \stackrel{\iota}{\hookrightarrow} \mathrm{X}$, qui se factorise donc en :

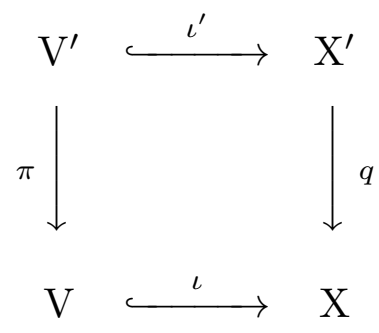

où $\iota^{\prime}$ est un plongement et $q$ une isogénie. Le morphisme induit $\mathrm{V}^{\prime} \rightarrow \mathrm{V} \times \mathrm{X}_{\mathrm{X}} \mathrm{X}^{\prime}$ est alors un plongement étale. Comme $\mathrm{V} \times \mathrm{X} \mathrm{X}^{\prime}$ est connexe (théorème 4.5), c'est un isomorphisme ([G1], Exp. I, cor. 5.2). Par (1.3), ceci montre l'injectivité du morphisme $\pi_{1}^{\text {alg }}(\mathrm{V}) \rightarrow \pi_{1}^{\text {alg }}(\mathrm{X})$; celui-ci est d'autre part surjectif par le corollaire 3.5.

Remarques 5.3. 1) J'ignore si la restriction sur les singularités de $\mathrm{V}$ est nécessaire.

2) Lorsque $\mathrm{V}$ est de plus normal, il ressort du corollaire et de (1.5) que le morphisme de restriction $\operatorname{Pic}^{0}(\mathrm{X}) \rightarrow \mathrm{Pic}^{0}(\mathrm{~V})$ est un isomorphisme. Si V est lisse, le corollaire 2.2 montre que la restriction $\operatorname{Pic}(\mathrm{X}) \rightarrow \operatorname{Pic}(\mathrm{V})$ est injective. Il serait intéressant de savoir si cette conclusion subsiste si l'on permet à $\mathrm{V}$ d'être singulier.

\section{Le problème de Zariski pour les variétés abéliennes}

Soient $\mathrm{X}$ une variété abélienne et $\mathrm{D}$ un diviseur de $\mathrm{X}$. Le problème de Zariski est l'étude du groupe fondamental algébrique $X-D$. En suivant la démarche de [F2], on va voir qu'il est possible de retrouver certains cas particuliers des résultats très généraux de $[\mathrm{N}]$.

On dira que le diviseur $\mathrm{D}$ de $\mathrm{X}$ est à croisements normaux en codimension 1 s'il existe un sous-schéma fermé $\mathrm{Z}$ de $\mathrm{D}$ de codimension 2 dans $\mathrm{D}$ tel que $\mathrm{D}-\mathrm{Z}$ soit un diviseur à croisements normaux dans $\mathrm{X}-\mathrm{Z}$. 
Théorème 6.1. - Soient $\mathrm{X}$ une variété abélienne de dimension $>1$ et $\mathrm{D}$ un diviseur de $\mathrm{X}$ à croisements normaux en codimension 1. On suppose qu'aucune composante de $\mathrm{D}$ n'est une sous-variété abélienne de $\mathrm{X}$. Alors le noyau du morphisme canonique $\pi_{1}^{\bmod }(\mathrm{X}-\mathrm{D}) \rightarrow \pi_{1}^{\mathrm{alg}}(\mathrm{X})$ est abélien.

Remarque 6.2. Il est clair que le résultat ne subsiste pas en général si $\mathrm{D}$ contient une sousvariété abélienne $\mathrm{K}$ de $\mathrm{X}$. Notons $\mathrm{E}$ la courbe elliptique $\mathrm{X} / \mathrm{K}$; on a alors des surjections :

$\operatorname{Ker}\left(\pi_{1}^{\mathrm{alg}}(\mathrm{X}-\mathrm{D}) \rightarrow \pi_{1}^{\mathrm{alg}}(\mathrm{X})\right) \rightarrow \operatorname{Ker}\left(\pi_{1}^{\mathrm{alg}}(\mathrm{X}-\mathrm{K}) \rightarrow \pi_{1}^{\mathrm{alg}}(\mathrm{X})\right) \rightarrow \operatorname{Ker}\left(\pi_{1}^{\mathrm{alg}}(\mathrm{E}-\{0\}) \rightarrow \pi_{1}^{\mathrm{alg}}(\mathrm{E})\right)$

Le groupe fondamental algébrique de $\mathrm{E}-\{0\}$ est isomorphe au complété profini de $\mathbf{Z} * \mathbf{Z}$, dont le noyau du morphisme canonique vers $\pi_{1}^{\text {alg }}(\mathrm{E}) \simeq \hat{\mathbf{Z}}$ n'est pas abélien.

Démonstration du théorème. Soient $\mathrm{V}$ une variété irréductible normale et $f: \mathrm{V} \rightarrow \mathrm{X}$ un revêtement Galoisien de groupe $\mathrm{G}$, dont le discriminant est contenu dans D. Il existe une factorisation $f: \mathrm{V} \stackrel{f^{\prime}}{\rightarrow} \mathrm{X}^{\prime} \stackrel{q}{\rightarrow} \mathrm{X}$, où $q$ est une isogénie et où $f^{\prime}$ est un revêtement Galoisien de groupe $\mathrm{G}^{\prime} \subset \mathrm{G}$, minimal au sens de (1.8). Le diviseur $\mathrm{D}^{\prime}=q^{-1}(\mathrm{D})$ de $\mathrm{X}^{\prime}$ est encore à croisements normaux en codimension 1. Soit B une composante irréductible de $\mathrm{D}^{\prime}$. Nous allons montrer que $f^{\prime-1}(\mathrm{~B})$ est irréductible.

Soit $\tilde{\mathrm{B}} \rightarrow \mathrm{B}$ la normalisation. On vérifie à l'aide du lemme d'Abhyankar ([G1], 3.6, Exp. $\mathrm{X})$ que $\left(\tilde{\mathrm{B}} \times_{\mathrm{X}^{\prime}} \mathrm{V}\right)_{\text {red }}$ est non singulier en codimension 1 . Comme $\mathrm{V}$ est normale, que $f^{\prime}$ est minimale et que $\mathrm{B}$ engendre $\mathrm{X}^{\prime}$, il résulte du théorème 4.5 que $\tilde{\mathrm{B}} \times_{\mathrm{X}^{\prime}} \mathrm{V}$ est connexe. Comme il est de plus de Cohen-Macaulay, il s'ensuit que ce schéma est irréductible ([H1]). Il en de même pour sa projection $f^{\prime-1}(\mathrm{~B})$ sur $\mathrm{V}$.

Les composantes de $\mathrm{D}^{\prime}$ se rencontrant deux à deux, il en est de même pour leurs images inverses dans V. Le lemme d'Abhyankar entraîne que les inerties correspondantes commutent deux à deux ([S]). Elles engendrent donc un sous-groupe abélien I de G' . Posons $\mathrm{X}^{\prime \prime}=\mathrm{V} / \mathrm{I}$. Le morphisme $f^{\prime}$ se factorise en $\mathrm{V} \rightarrow \mathrm{X}^{\prime \prime} \stackrel{f^{\prime \prime}}{\rightarrow} \mathrm{X}^{\prime}$ et $f^{\prime \prime}$ est alors non ramifié en codimension 1 , donc non ramifié d'après le théorème de pureté.

Posons $\mathrm{D}^{\prime \prime}=f^{\prime \prime-1}\left(\mathrm{D}^{\prime}\right)$. On a un diagramme commutatif :

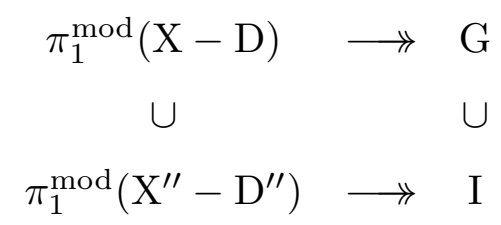

D'autre part, $\pi_{1}^{\bmod }\left(\mathrm{X}^{\prime \prime}-\mathrm{D}^{\prime \prime}\right)$ contient le noyau $\mathrm{N}$ du morphisme canonique $\pi_{1}^{\bmod }(\mathrm{X}-\mathrm{D}) \rightarrow \pi_{1}^{\mathrm{alg}}(\mathrm{X}) \quad([\mathrm{G} 1]$, cor. 6.7, Exp. V). Il s'ensuit que l'image de $\mathrm{N}$ dans $\mathrm{G}$ est contenue dans I, donc est abélienne. 
Nous avons donc montré que pour tout sous-groupe ouvert distingué $\Gamma$ de $\pi_{1}^{\bmod }(\mathrm{X}-\mathrm{D})$, le groupe $\mathrm{N} /(\mathrm{N} \cap \Gamma)$ est abélien. Comme $\mathrm{N}$ est fermé, il s'identifie à $\varliminf_{\Gamma} \mathrm{N} /(\mathrm{N} \cap \Gamma)\left([\mathrm{Bo}], \mathrm{III}, \S 7, \mathrm{n}^{\mathrm{o}} 2\right.$, prop. 3). C'est donc un groupe abélien.

Lorsque $k=\mathbf{C}$, Nori montre dans $[\mathrm{N}]$ que le noyau du morphisme $\pi_{1}(\mathrm{X}-\mathrm{D}) \rightarrow \pi_{1}(\mathrm{X})$ entre groupes fondamentaux topologiques est abélien de type fini, et que son centralisateur est d'indice fini. On peut préciser son résultat de la façon suivante.

Proposition 6.3. - Sous les hypothèses du théorème, et lorsque $k=\mathbf{C}$, le noyau $\mathrm{N} d u$ morphisme canonique $\pi_{1}(\mathrm{X}-\mathrm{D}) \rightarrow \pi_{1}(\mathrm{X})$ est abélien libre de type fini.

Remarques 6.4. 1) Soient $\tilde{\mathrm{D}}_{1}, \ldots, \tilde{\mathrm{D}}_{r}$ les composantes irréductibles de la normalisation de $\mathrm{D}$, et $\mathrm{K}_{i}$ le noyau du morphisme $\operatorname{Pic}^{0}(\mathrm{X}) \rightarrow \operatorname{Pic}^{0}\left(\tilde{\mathrm{D}}_{i}\right)$. Alors le rang de $\mathrm{N}$ est $\sum_{i=1}^{r} \operatorname{Card}\left(\mathrm{K}_{i}\right)$.

2) Lorsque les composantes du diviseur $\mathrm{D}$ sont de plus normales, le rang de $\mathrm{N}$ est le nombre $r$ de composantes irréductibles de $\mathrm{D}$, et Nori montre que l'extension :

$$
0 \rightarrow \mathbf{Z}^{r} \rightarrow \pi_{1}(\mathrm{X}-\mathrm{D}) \rightarrow \pi_{1}(\mathrm{X}) \rightarrow 0
$$

est centrale et que sa classe correspond au $r$-uplet des classes fondamentales des composantes de $\mathrm{D}$ via l'isomorphisme $\mathrm{H}^{2}\left(\pi_{1}(\mathrm{X}), \mathbf{Z}^{r}\right) \simeq \mathrm{H}^{2}(\mathrm{X}, \mathbf{Z})^{r}$.

Démonstration de la proposition. Soit $k$ le nombre maximum de composantes irréductibles de l'image inverse de $\mathrm{D}$ par une isogénie $\mathrm{X}^{\prime} \rightarrow \mathrm{X}$. On vérifie que ce nombre est fini et qu'il se calcule comme dans la remarque ci-dessus. Les inerties étant cycliques, la démonstration du théorème montre que tout quotient fini de $\mathrm{N}$ (donc aussi $\mathrm{N}$ ) peut être engendré par $k$ éléments. Soit $q: \mathrm{X}^{\prime} \rightarrow \mathrm{X}$ une isogénie telle que $\mathrm{D}^{\prime}=q^{-1}(\mathrm{D})$ ait $k$ composantes. On vérifie que $\mathrm{N}$ est isomorphe au noyau $\mathrm{N}^{\prime}$ du morphisme $\pi_{1}\left(\mathrm{X}^{\prime}-\mathrm{D}^{\prime}\right) \rightarrow \pi_{1}\left(\mathrm{X}^{\prime}\right)$. D'autre part, il existe une surjection de $\mathrm{N}^{\prime}$ sur le noyau de $\mathrm{H}_{1}\left(\mathrm{X}^{\prime}-\mathrm{D}^{\prime}, \mathbf{Z}\right) \rightarrow \mathrm{H}_{1}\left(\mathrm{X}^{\prime}, \mathbf{Z}\right)$. Un argument classique de dualité montre que ce dernier est isomorphe au conoyau du morphisme $\mathrm{H}_{2}\left(\mathrm{X}^{\prime}, \mathbf{Z}\right) \rightarrow \mathrm{H}^{2 \operatorname{dim}\left(\mathrm{D}^{\prime}\right)}\left(\mathrm{D}^{\prime}, \mathbf{Z}\right) \simeq \mathbf{Z}^{k}$, qui envoie $\gamma \operatorname{sur}\left(d_{1}(\gamma), \ldots, d_{k}(\gamma)\right)$, où $d_{1}, \ldots, d_{k}$ sont les classes fondamentales des composantes de $\mathrm{D}^{\prime}$. Soit $\mathrm{M}: \mathrm{X}^{\prime} \rightarrow \mathrm{X}^{\prime}$ la multiplication par un entier $m$. Posons $\mathrm{D}^{\prime \prime}=\mathrm{M}^{-1}\left(\mathrm{D}^{\prime}\right)$. De nouveau, $\mathrm{N}^{\prime}$ est isomorphe au noyau $\mathrm{N}^{\prime \prime}$ du morphisme $\pi_{1}\left(\mathrm{X}^{\prime \prime}-\mathrm{D}^{\prime \prime}\right) \rightarrow \pi_{1}\left(\mathrm{X}^{\prime \prime}\right)$ et les composantes de $\mathrm{D}^{\prime \prime}$ sont les images inverses de celles de $\mathrm{D}^{\prime}$, donc leurs classes sont divisibles par $m^{2}$. Il ressort de ce qui précède qu'il existe une surjection de $\mathrm{N}^{\prime \prime}$ sur $\mathbf{Z}^{k} /\left(m^{2}, \ldots, m^{2}\right)$. Ceci étant vrai pour tout $m$, il existe une surjection de $\mathrm{N}$ sur $\mathbf{Z}^{k}$. Comme $\mathrm{N}$ est engendré par $k$ éléments, il est isomorphe à $\mathbf{Z}^{k}$.

\section{Revêtements des sous-variétés des variétés abéliennes}

Suivant toujours [FL], on s'intéresse maintenant aux variétés qui sont revêtements finis ramifiés de petit degré d'une sous-variété d'une variété abélienne simple. Pour énoncer notre résultat, dont la démonstration est calquée sur celle du théorème principal de [GL], nous 
utiliserons la notion de degré local d'un morphisme fini $f: \mathrm{V} \rightarrow \mathrm{W}$ en un point $v \in \mathrm{V}$, telle qu'elle est définie dans [GL] et [Mu2]. Intuitivement, c'est le nombre de feuillets de $f$ qui se rejoignent en $v$. On le note $e_{f}(v)$.

Théorème 7.1. - Soient $\mathrm{X}$ une variété abélienne simple, $\mathrm{W}$ une sous-variété lisse de $\mathrm{X}$, $\mathrm{V}$ une variété irréductible normale et $f: \mathrm{V} \rightarrow \mathrm{W}$ un revêtement fini de degré $d$. On suppose le morphisme composé $\mathrm{V} \rightarrow \mathrm{W} \hookrightarrow \mathrm{X}$ est minimal au sens de (1.8). Alors il existe un point $v \in \mathrm{V}$ tel que :

$$
e_{f}(v) \geq \min (d, 2 \operatorname{dim}(\mathrm{W})-\operatorname{dim}(\mathrm{X})+1) .
$$

Démonstration. Pour tout entier $l$, l'ensemble :

$$
\mathrm{R}_{l}=\left\{v \in \mathrm{V} \mid e_{f}(v)>l\right\}
$$

est fermé dans V ([GL], lemma 1). De plus, comme W est lisse, le théorème 2.2 de [L2] (cf. aussi [GL], p. 58), entraîne que $\mathrm{R}_{l}$ est soit vide, soit partout de codimension $\leq l$ dans $\mathrm{V}$. Il s'agit de montrer que $\mathrm{R}_{l}$ est non vide pour $l \leq \min (d-1,2 \operatorname{dim}(\mathrm{W})-\operatorname{dim}(\mathrm{X}))$. Nous procédons par récurrence sur $l$. On a $R_{0}=\mathrm{V}$; on suppose que $\mathrm{R}_{l-1}$ est non vide, et on en choisit une composante irréductible $\mathrm{R}$. La projection $\mathrm{V} \times_{\mathrm{X}} \mathrm{R} \rightarrow \mathrm{R}$ est finie, de sorte que la diagonale $\Delta_{\mathrm{R}}$ est une composante irréductible de $\mathrm{V} \times_{\mathrm{X}} \mathrm{R}$. Comme :

$$
\operatorname{dim}(\mathrm{R}) \geq \operatorname{dim}(\mathrm{V})-(l-1) \geq \operatorname{dim}(\mathrm{V})-2 \operatorname{dim}(\mathrm{W})+\operatorname{dim}(\mathrm{X})+1>\operatorname{dim}(\mathrm{X})-\operatorname{dim}(\mathrm{V})
$$

le théorème 4.5 entraîne que $\mathrm{V} \times_{\mathrm{X}} \mathrm{R}$ est connexe. Si $\mathrm{V} \times_{\mathrm{X}} \mathrm{R}=\Delta_{\mathrm{R}}$, alors $\mathrm{R} \subset \mathrm{R}_{d-1} \subset \mathrm{R}_{l}$, ce qui permet de conclure. Sinon, il existe une composante irréductible $T$ de $V \times_{X} R$ distincte de $\Delta_{\mathrm{R}}$, qui rencontre la diagonale. Comme dans [GL], p. 57, on conclut que pour tout point $(v, v)$ de $\mathrm{T} \cap \Delta_{\mathrm{R}}$, on a $e_{f}(v)>l$, c'est-à-dire $v \in \mathrm{R}_{l}$.

Comme dans [GL], on en déduit :

Corollaire 7.2. - Soient $\mathrm{X}$ une variété abélienne simple, W une sous-variété lisse de $\mathrm{X}, \mathrm{V}$ une variété irréductible unibranche et $f: \mathrm{V} \rightarrow \mathrm{W}$ un revêtement fini de degré $d \leq 2 \operatorname{dim}(\mathrm{W})-\operatorname{dim}(\mathrm{X})$. Alors, le morphisme induit $\pi_{1}^{\mathrm{alg}}(\mathrm{V}) \rightarrow \pi_{1}^{\mathrm{alg}}(\mathrm{X})$ est injectif et son image est isomorphe $\grave{a} \pi_{1}^{\mathrm{alg}}(\mathrm{X})$.

Démonstration. Par (1.6), on peut supposer $\mathrm{V}$ normale. Toute isogénie $\mathrm{X}^{\prime} \rightarrow \mathrm{X}$ induit une injection $\pi_{1}^{\text {alg }}\left(\mathrm{X}^{\prime}\right) \rightarrow \pi_{1}^{\text {alg }}(\mathrm{X})$ (1.4) dont l'image est isomorphe à $\pi_{1}^{\text {alg }}(\mathrm{X})(1.7)$. On peut donc supposer que le morphisme composé $g: \mathrm{V} \rightarrow \mathrm{W} \hookrightarrow \mathrm{X}$ est minimal. Soit $\pi: \mathrm{V}^{\prime} \rightarrow \mathrm{V}$ un revêtement étale connexe; $\mathrm{V}$ étant irréductible et normale, il en est de même de $\mathrm{V}^{\prime}$ (1.6). Soit $\mathrm{V}^{\prime} \stackrel{g^{\prime}}{\rightarrow} \mathrm{X}^{\prime} \stackrel{p}{\rightarrow} \mathrm{X}$ une factorisation de $g \pi$ avec $g^{\prime}$ minimal (1.8). Posons $\mathrm{W}^{\prime}=g^{\prime}\left(\mathrm{V}^{\prime}\right)$, et notons $f^{\prime}: \mathrm{V}^{\prime} \rightarrow \mathrm{W}^{\prime}$ le revêtement induit et $d^{\prime}$ son degré. Le théorème s'applique à $f^{\prime}$ et il existe $v^{\prime} \in \mathrm{V}^{\prime}$ tel que :

$$
e_{f^{\prime}}\left(v^{\prime}\right) \geq \min \left(d^{\prime}, 2 \operatorname{dim}\left(\mathrm{W}^{\prime}\right)-\operatorname{dim}\left(\mathrm{X}^{\prime}\right)+1\right)
$$


Comme $p$ et $\pi$ sont étales et que $d$ est le degré de $f$, on a :

$$
e_{f^{\prime}}\left(v^{\prime}\right)=e_{p f^{\prime}}\left(v^{\prime}\right)=e_{f \pi}\left(v^{\prime}\right)=e_{f}\left(\pi\left(v^{\prime}\right)\right) \leq d
$$

Comme $d \leq 2 \operatorname{dim}(\mathrm{W})-\operatorname{dim}(\mathrm{X})$, il s'ensuit que $d \geq d^{\prime}$, donc que :

$$
\operatorname{deg}(\pi) \leq \operatorname{deg}\left(p_{\mid \mathrm{W}^{\prime}}\right) \leq \operatorname{deg}(p)
$$

Or $\pi$ se factorise à travers le morphisme étale $\mathrm{V} \times_{\mathrm{X}} \mathrm{X}^{\prime} \rightarrow \mathrm{V}$, qui est de même degré que $p$. Comme $\mathrm{V} \times_{\mathrm{X}} \mathrm{X}^{\prime}$ est connexe (théorème 4.5) donc irréductible (1.6), le morphisme induit $\mathrm{V}^{\prime} \rightarrow \mathrm{V} \times{ }_{\mathrm{X}} \mathrm{X}^{\prime}$ est un isomorphisme. Par (1.3), cela démontre le corollaire.

Remarques 7.3. 1) La conclusion du corollaire ne subsiste en général pas sans hypothèse sur les singularités de V, comme le montre la construction de [FL], note (2), p. 56.

2) On a besoin de la lissité de $W$ uniquement pour pouvoir appliquer le résultat de Lazarsfeld. Plus précisément, il faut savoir que les lieux $\mathrm{R}_{l}$ sont de codimension $\leq l$ pour $l<2 \operatorname{dim}(\mathrm{W})-\operatorname{dim}(\mathrm{X})<d$. Un théorème de pureté de Grothendieck ([G3], Exp. X, th. 3.4) dit que c'est le cas pour $\mathrm{V}$ normal, W localement intersection complète, $l=1$ et $\operatorname{codim}(\operatorname{Sing}(\mathrm{W})) \geq 3$. Cela suggère que le corollaire devrait s'étendre au cas W localement intersection complète et $\operatorname{dim}(\operatorname{Sing}(\mathrm{W}))<\operatorname{codim}(\mathrm{W})$.

Corollaire 7.4. - Soient $\mathrm{X}$ une variété abélienne simple, $\mathrm{W}$ une sous-variété lisse de $\mathrm{X}$, $\mathrm{V}$ une variété irréductible et $f: \mathrm{V} \rightarrow \mathrm{W}$ un revêtement fini de degré $d$. Si $\mathrm{V}_{1}$ et $\mathrm{V}_{2}$ sont deux sous-variétés de $\mathrm{V}$ telles que:

$$
\operatorname{dim}\left(\mathrm{V}_{1}\right)+\operatorname{dim}\left(\mathrm{V}_{2}\right) \geq 2 \operatorname{dim}(\mathrm{X})-\operatorname{dim}(\mathrm{V})+d-1
$$

alors $\mathrm{V}_{1} \cap \mathrm{V}_{2} \neq \emptyset$. En particulier, tout morphisme de $\mathrm{V}$ dans une variété de dimension $\leq 3 \operatorname{dim}(\mathrm{V})-2 \operatorname{dim}(\mathrm{X})-d$ est constant.

Démonstration du corollaire. La démonstration suit [L1], remarque 2.3. On peut supposer $\mathrm{V}$ normale et $d \leq 2 \operatorname{dim}(\mathrm{W})-\operatorname{dim}(\mathrm{X})$. Par le théorème 7.1 et le résultat de [L2] utilisé plus haut, il existe une sous-variété $\mathrm{R}$ de $\mathrm{V}$ de codimension $\leq d-1$ telle que $f$ soit bijective audessus de $f(\mathrm{R})$. La remarque 3.2.2) entraîne alors que $f\left(\mathrm{~V}_{1}\right), f\left(\mathrm{~V}_{2}\right)$ et $f(\mathrm{R})$ se rencontrent, donc aussi $V_{1}, V_{2}$ et $R$. La dernière assertion en résulte immédiatement $:$ si $V \rightarrow T$ est surjectif et si $\operatorname{dim}(\mathrm{T})>0$, il suffit de prendre pour $\mathrm{V}_{1}$ l'image inverse d'un diviseur et pour $\mathrm{V}_{2}$ l'image inverse d'un point hors de ce diviseur.

La démonstration du théorème suivant suit celle d'un résultat analogue de Lazarsfeld ([F3], th., p. 151).

Théorème 7.5. - Soient $\mathrm{X}$ une variété abélienne simple, $\mathrm{V}$ une variété irréductible unibranche et $f: \mathrm{V} \rightarrow \mathrm{X}$ un morphisme. On suppose qu'il existe une sous-variété $\mathrm{Z}$ de 
$f(\mathrm{~V})$ vérifiant $\operatorname{dim}(\mathrm{Z})>\operatorname{codim}(f(\mathrm{~V}))$ telle que le morphisme induit $f^{-1}(\mathrm{Z}) \rightarrow \mathrm{Z}$ soit une bijection ensembliste. Alors, le morphisme induit $\pi_{1}^{\text {alg }}(\mathrm{V}) \rightarrow \pi_{1}^{\text {alg }}(\mathrm{X})$ est injectif et son image est isomorphe $\grave{a} \pi_{1}^{\text {alg }}(\mathrm{X})$.

Remarque 7.6. Le théorème s'applique en particulier aux revêtements cycliques des sousvariétés de $\mathrm{X}$ de dimension $\geq 1+\frac{1}{2} \operatorname{dim}(\mathrm{X})$.

Démonstration. On peut comme d'habitude supposer $f$ minimal, et $Z$ intègre. Soit $\tilde{Z}$ la normalisation de $\mathrm{Z}$; on pose $\mathrm{Z}^{\prime}=\tilde{\mathrm{Z}} \times \mathrm{X} \mathrm{V}$. Nos hypothèses entraînent que le morphisme induit $\mathrm{Z}_{\text {red }}^{\prime} \rightarrow \tilde{Z}$ est fini et birationnel; c'est donc un isomorphisme par le théorème principal de Zariski ([GD2], 8.12.10.1). Le morphisme $\pi_{1}^{\text {alg }}\left(\mathrm{Z}_{\text {red }}^{\prime}\right) \rightarrow \pi_{1}^{\text {alg }}\left(\mathrm{Z}^{\prime}\right)$ étant surjectif (1.2), l'intersection de l'image du morphisme :

$$
\pi_{1}^{\mathrm{alg}}\left(\mathrm{Z}^{\prime}\right) \rightarrow \pi_{1}^{\mathrm{alg}}(\tilde{\mathrm{Z}} \times \mathrm{V}) \simeq \pi_{1}^{\mathrm{alg}}(\tilde{\mathrm{Z}}) \times \pi_{1}^{\mathrm{alg}}(\mathrm{V})
$$

avec $\{1\} \times \pi_{1}^{\text {alg }}(\mathrm{V})$ est réduite à $\{(1,1)\}$. Comme cette image est le noyau du morphisme canonique $\pi_{1}^{\text {alg }}(\tilde{\mathrm{Z}} \times \mathrm{V}) \rightarrow \pi_{1}^{\text {alg }}(\mathrm{X})$ (théorème 4.5 ), il s'ensuit que le morphisme $\pi_{1}^{\text {alg }}(\mathrm{V}) \rightarrow \pi_{1}^{\text {alg }}(\mathrm{X})$ est injectif. Il est d'autre part surjectif par le corollaire 4.7, ce qui conclut la démonstration.

On termine ce chapitre avec la démonstration d'un résultat analogue à un théorème de Ein sur les revêtements des espaces projectifs. Elle utilise des résultats puissants de Kawamata, Kollár et Mori, mais je pense qu'il devrait exister une démonstration commune (plus simple) à base de connexité.

Théorème 7.7. - Soient $\mathrm{X}$ est une variété abélienne simple, $\mathrm{V}$ une variété lisse et $f: \mathrm{V} \rightarrow \mathrm{X}$ un revêtement fini ramifié. Alors la ramification de $f$, c'est-à-dire le faisceau canonique de $\mathrm{V}$, est ample.

Remarque 7.8. Le théorème de connexité 4.5 entraîne que la ramification de $f$ rencontre toute courbe de $\mathrm{V}$.

Démonstration. Par [K1], theorem 13, il existe une sous-variété abélienne $\mathrm{Y}$ de $\mathrm{X}$, un morphisme fini $\mathrm{W} \rightarrow \mathrm{X} / \mathrm{Y}$ avec $\kappa(\mathrm{V})=\operatorname{dim}(\mathrm{W})$, et des revêtements étales $\tilde{\mathrm{Y}} \rightarrow \mathrm{Y}$ et $\tilde{\mathrm{Y}} \times \mathrm{W} \rightarrow \mathrm{V}$. Comme $\mathrm{X}$ est simple, on a soit $\mathrm{Y}=\mathrm{X}$, auquel cas $f$ est étale, ce qui contredit l'hypothèse; soit $\mathrm{Y}=0$, et $\kappa(\mathrm{V})=\operatorname{dim}(\mathrm{V})$, de sorte que $\mathrm{V}$ est de type général. Le théorème découle alors du lemme suivant.

Lemme 7.9. - Soient $\mathrm{V}$ une variété projective lisse de type général. Alors soit $\mathrm{K}_{\mathrm{V}}$ est ample, soit $\mathrm{V}$ contient une courbe rationnelle.

Démonstration. On suit $[\mathrm{Ko}]$. Par $[\mathrm{M}]$, theorem 1.4, soit V contient une courbe rationnelle, soit $\mathrm{K}_{\mathrm{V}}$ est nef. Dans le second cas, il découle de [CKM], (9.3), que le système linéaire $\left|m \mathrm{~K}_{\mathrm{V}}\right|$ est sans point base pour $m \gg 0$, donc définit un morphisme $\phi$ génériquement fini sur son image. $\mathrm{Si} \phi$ est fini, $\mathrm{K}_{\mathrm{V}}$ est ample; sinon, il contracte une courbe $\mathrm{C}$, qui vérifie alors 
$\mathrm{C} \cdot \mathrm{K}_{\mathrm{V}}=0$. Soit $\mathrm{H}$ un diviseur très ample sur $\mathrm{V}$; son image $\phi(\mathrm{H})$ est contenue dans une hypersurface de degré $r$, de sorte qu'il existe un diviseur effectif $\mathrm{E}$ tel que $r m \mathrm{~K}_{\mathrm{V}} \equiv \mathrm{H}+\mathrm{E}$. On a donc $\mathrm{C} \cdot \mathrm{E}<0$. Il existe alors $\epsilon$ rationnel positif tel que le diviseur $\mathrm{K}_{\mathrm{V}}+\epsilon \mathrm{E}$ soit log-canonique $([\mathrm{KMM}]$, lemma $0-2-15)$. Comme $\left(\mathrm{K}_{\mathrm{V}}+\epsilon \mathrm{E}\right) \cdot \mathrm{C}<0$, il n'est pas nef, et le théorème du cône (loc.cit., theorem 4-2-1) entraîne qu'il existe une raie extrêmale et un morphisme de contraction (loc.cit., theorem 3-2-1) $g: \mathrm{V} \rightarrow \mathrm{W}$, auquel on peut appliquer [K2], theorem 1 : le lieu des points où $g$ n'est pas un isomorphisme est recouvert par des courbes rationnelles.

\section{Conjecture}

Une démonstration de la conjecture suivante permettrait d'utiliser les résultats de Lazarsfeld ([L1], th. 2.1, prop. 3.1), et entraînerait en particulier notre théorème 7.1 sur les indices de ramification.

Conjecture 8.1. Soient $\mathrm{X}$ est une variété abélienne simple, $\mathrm{V}$ une variété lisse et $f: \mathrm{V} \rightarrow \mathrm{X}$ un revêtement fini, minimal au sens de (1.8). On définit un faisceau localement libre $\mathrm{E}$ sur $\mathrm{X}$ comme le dual du noyau de la trace $\operatorname{Tr}_{\mathrm{V} / \mathrm{X}}: f_{*} \mathcal{O}_{\mathrm{V}} \longrightarrow \mathcal{O}_{\mathrm{X}}$. Alors $\mathrm{E}$ est ample.

La réponse est affirmative si $\mathrm{X}$ est une courbe elliptique. En effet, par un théorème de Hartshorne ([H2]), il suffit de démontrer que tout faisceau inversible L quotient de $\mathrm{E}$ est de degré $>0$. Soit L un tel faisceau; on a alors :

$$
0<h^{0}\left(\mathrm{X}, \mathrm{E}^{\vee} \otimes \mathrm{L}\right)=h^{0}\left(\mathrm{X}, f_{*} \mathcal{O}_{\mathrm{V}} \otimes \mathrm{L}\right)-h^{0}(\mathrm{X}, \mathrm{L})=h^{0}\left(\mathrm{~V}, f^{*} \mathrm{~L}\right)-h^{0}(\mathrm{X}, \mathrm{L})
$$

Cela entraîne en particulier $h^{0}\left(\mathrm{~V}, f^{*} \mathrm{~L}\right)>0$. Si $\operatorname{deg}(\mathrm{L}) \leq 0$, alors $\operatorname{deg}\left(f^{*} \mathrm{~L}\right) \leq 0$ et $f^{*} \mathrm{~L}$ est donc trivial. On a alors $h^{0}\left(\mathrm{~V}, f^{*} \mathrm{~L}\right)=1$ et $h^{0}(\mathrm{X}, \mathrm{L})=0$, donc $\mathrm{L} \not \mathcal{O}_{\mathrm{X}}$, ce qui contredit l'injectivité de $f^{*}$. On a donc démontré que $\operatorname{deg}(\mathrm{L})>0$.

On peut voir cette conjecture comme une forme forte d'un théorème de connexité : supposons E ample; si $\mathrm{W}$ est une variété irréductible de dimension $>0$ et si $g: \mathrm{W} \rightarrow \mathrm{X}$ est un morphisme fini, alors $g^{*} \mathrm{E}$ est ample, donc $\mathrm{H}^{0}\left(\mathrm{~W}, g^{*} \mathrm{E}^{\vee}\right)=0$. Si on pose $\mathrm{W}^{\prime}=\mathrm{V} \times_{\mathrm{X}} \mathrm{W}$, cela se traduit par $h^{0}\left(\mathrm{~W}^{\prime}, \mathcal{O}_{\mathrm{W}^{\prime}}\right)=h^{0}\left(\mathrm{~W}, \mathcal{O}_{\mathrm{W}}\right)=1$, qui est évidemment plus fort que la connexité de $\mathrm{W}^{\prime}$ (assurée par le théorème 4.5).

\section{REFERENCES}

[B1] Barth, W., Fortsetzung meromorpher Funktionen in Tori und Komplexprojektiven Räumen, Invent. Math 5 (1968), 42-62.

[B2] Barth, W., Verallgemeinerung des bertinischen Theorems in abelschen Mannigfaltigkeiten, Ann. Sc. Norm. Sup. Pisa, Serie IV, 23 (1969), 317-330. 
[Bo] Bourbaki,N., Topologie Générale, Hermann, 1960.

[CKM] Clemens, H., Kollár, J., Mori, S., Higher Dimensional Complex Geometry, Astérisque 166.

[F1] Fulton, W., On the Topology of Algebraic Varieties, in Algebraic Geometry Bowdoin 1985, Proceedings of Symposia in Pure Mathematics 46, Part 1, 1987.

[F2] Fulton, W., On the Fundamental Group of the Complement of a Node Curve, Ann. Math. 11 (1980), 407-409.

[F3] Fulton, W., On Nodal Curves, in Algebraic Geometry - Open Problems, Proceedings, Ravello 1982, Springer Lecture Notes 977.

[FH] Fulton, W., Hansen, J. A Connectedness Theorem for Projective Varieties, with Applications to Intersections and Singularities of Mappings, Ann. of Math. 110 (1979), 159-166.

[FL] Fulton, W., Lazarsfeld, R., Connectivity and its Applications in Algebraic Geometry, in Algebraic Geometry, Proceedings of the Midwest Algebraic Geometry Conference, Chicago 1980, Springer Lecture Notes 862.

[GL] Gaffney, T., Lazarsfeld, R., On the Ramification of Branched Coverings of $\mathbf{P}^{n}$, Invent. Math. 59 (1980), 53-58.

[G1] Grothendieck, A., Revêtements Etales et Groupe Fondamental, S.G.A. 1, Springer Lecture Notes 224.

[G2] Grothendieck, A., Fondements de la Géométrie Algébrique, Séminaire Bourbaki 19571962.

[G3] Grothendieck, A., Cohomologie locale des faisceaux cohérents et théorèmes de Lefschetz locaux et globaux, S.G.A. 2, North Holland, Amsterdam, 1968.

[GD1] Grothendieck, A., Dieudonné, J., Eléments de Géométrie Algébrique IV, 4, Publ. Math. I.H.E.S. 32, 1967.

[GD2] Grothendieck, A., Dieudonné, J., Eléments de Géométrie Algébrique IV, 3, Publ. Math. I.H.E.S. 28, 1966.

[H1] Hartshorne, R., Complete Intersections and Connectedness, Amer. J. Math. 84 (1962), 497-508.

[H2] Hartshorne, R., Ample vector bundles on curves, Nagoya Math. J. 43 (1971), 73-89.

[KMM] Kawamata, Y., Matsuda, K., Matsuki, K., Introduction to the Minimal Model Problem, in Algebraic Geometry Sendai 1985, Oda, T. editor, Adv. Stud. Pure Math. 10, Tokyo, 1987.

[K1] Kawamata, Y., Characterization of Abelian Varieties, Comp. Math. 43 (1981), 253-276. 
[K2] Kawamata, Y., On the Length of an Extremal Rational Curve, Inv. Math. 105 (1991), 609-611.

[Ko] Kollár, J., Shafarevich Maps and Automorphic Forms, preprint.

[L1] Lazarsfeld, R., A Barth-Type Theorem for Branched Coverings of Projective Space, Math. Ann. 249 (1980), 153-162.

[L2] Lazarsfeld, R., Ph.D. thesis, Brown University, June 1980.

[M] Mori, S., Threefolds whose Canonical Bundles are not Numerically Effective, Ann. Math. 116 (1982), 133-176.

[Mu1] Mumford, D., Abelian Varieties, Oxford University Press, 1974.

[Mu2] Mumford, D., Algebraic Geometry I. Complex Projective Varieties, Grundlehren der mathematischen Wissenschaften 221, Springer Verlag, 1976.

[N] Nori, M., Zariski's conjecture and related problems, Ann. Sci. Ecole Norm. Sup. 16 (1983), $305-344$.

[S] Serre, J.-P., Revêtements ramifiés du plan projectif (d'après S. Abhyankar), Séminaire Bourbaki, Exp. 204, 1960.

[So] Sommese, A., Complex Subspaces of Homogeneous Complex Manifolds II. Homotopy Results, Nagoya Math. J. 86 (1982), 101-129.

[Z] Zariski, O., On the Purity of the Branch Locus of Algebraic Functions, Proc. Nat. Acad. Sci. 44 (1958), 791-796. 